\title{
MATCHING OF HIGH RESOLUTION OPTICAL DATA TO A SHADED DEM
}

\author{
Mathias Schneider, Peter Reinartz \\ German Aerospace Center (DLR), Remote Sensing Technology Institute, D-82234 Wessling, \\ Germany
}

\begin{abstract}
One of the first essential steps in the analysis of satellite imagery is the orthorectification of the images. Orthorectification without ground control points (GCPs) using only the ephemeris and attitude data provided by the satellite operator provides an absolute accuracy of about $20 \mathrm{~m}$ to $1 \mathrm{~km}$ (depending on the satellite), which can be improved by measuring precise GCPs. In this paper, a method to obtain GCPs from an existing digital elevation model (DEM) is described and assessed. Since at least the SRTM DEM is available worldwide, DEMs could serve as a valuable additional source for the generation of GCPs. Furthermore, several planned and ongoing missions will increase the availability and accuracy of DEMs or stereo imagery respectively, e.g. ALOS, Tandem-X, etc.
\end{abstract}

Index Terms - Orthoimages, DEM, Ground Control, Matching, Improvement of Orientation

\section{INTRODUCTION}

Normally, satellite data are delivered geocoded with varying accuracy. Orthorectification without ground control points (GCPs) using only the ephemeris and attitude data provided by the satellite operator provides an absolute accuracy of about $20 \mathrm{~m}$ to $1 \mathrm{~km}$ (depending on the satellite). For most applications like change detection this accuracy is not sufficient. For this purpose, accuracy in subpixel range is necessary. The orthorectification accuracy can be improved by measuring precise GCPs. However, often there is no possibility to measure precise GCPs in situ or through precise maps. An alternative method to obtain GCPs is a matching of the satellite image to a previously orthorectified image [8]. If none of these reference data are available, no correction can be made. In this paper, a method to obtain GCPs from an existing digital elevation model (DEM) is described and assessed. Since at least the SRTM DEM is available worldwide, DEMs can serve as a valuable additional source for the generation of GCPs. Furthermore, several planned and ongoing missions will increase the availability and accuracy of DEMs or stereo imagery respectively, e.g. ALOS, Tandem-X, etc.
In the first part of the paper, the method is described: From the existing DEM a shaded model is generated. Therefore, the same viewing angle as in the satellite image is used as well as the same sun incidence and elevation angles as during the image acquisition. This shaded model is then used as reference image for a hierarchical intensity based matching as described in [1] and the resulting matching points are used as GCPs. A similar approach is presented in [2], where - in contrast to this paper - a frequency based matching was used to extract GCPs. Additionally to the intensity based matching, in this paper also a mutual information based matching is performed on the data.

In the second part of the paper, an analysis of the performed tests is made. DEMs from different sources and different test areas are used. For a test area located in Yemen three different DEMs are available. Additional to the SRTM-DEM, a DEM is calculated from an IKONOS stereo pair and another DEM is calculated from an ALOS/PRISM triplet. The feasibility of the method is demonstrated depending on the source and resolution of the DEMs and the used matching algorithm.

It is shown that the presented method can provide a very useful additional source of GCPs for orthorectification purposes. Since the absolute accuracy of the GCPs depends on the accuracy of the used DEM, absolute subpixel or even pixel accuracy can not be reached in case of very high resolution images. However, a sufficient accurate coregistration of different images to a DEM can be realized which is necessary e.g. for change detection.

\section{TEST AREA AND DATA}

Our test area is located near Kaukaban, Yemen. The area is terraced and, besides some agricultural areas, with sparse vegetation which makes it a promising test area for our purposes. Three different DEM sources were available: The SRTMDEM, an ALOS PRISM image triplet and an IKONOS stereo pair. The SRTM-DEM is available worldwide between $56^{\circ}$ South and $60^{\circ}$ North with a resolution of 1 or 3 arcseconds respectively. The PRISM instrument on the Japanese ALOS satellite provides panchromatic forward, nadir and backward views of the area with a resolution of 2.5 
m. The imagery was recorded on March 24, 2008. A DEM is calculated from the image triplet as described in [3]. The IKONOS stereo pair was recorded on March 9, 2007 with a stereo angle of approximately $6^{\circ}$. A DEM is calculated as described e.g. in [4]. One of the images from the IKONOS stereo pair was used for the matching.

\section{METHOD}

\subsection{Data Preparation}

After the ALOS and the IKONOS DEM have been created, all three DEMS were resampled to a $10 \mathrm{~m}$ resolution and a shaded model was created. To reach a maximum of similarity between image and DEM, the shaded model is calculated by trying to simulate the conditions that were prevailing during the image acquisition. The same sun elevation and azimuth angles as were prevailing during the acquisition of the IKONOS image were used in the calculation of the model. Figure 1 shows the shaded model of the DEM created from the IKONOS stereo pair.

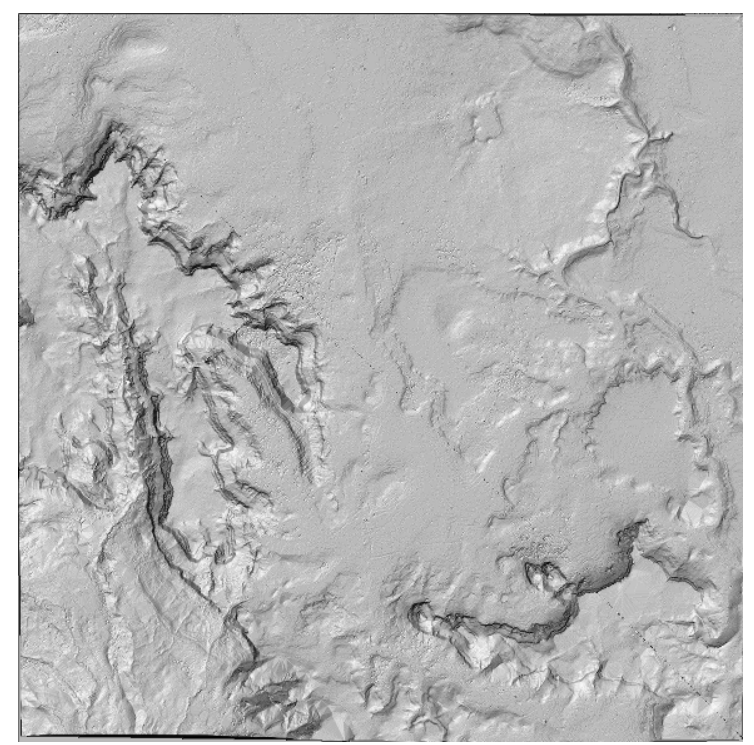

Figure 1: Shaded model of the DEM generated from IKONOS stereo pair with same sun elevation and azimuth angles as during the acquisition of the IKONOS image.

In order to improve the matching results, the shaded models were then resampled to a $1 \mathrm{~m}$ resolution and the IKONOS image was filtered with a median filter.

\subsection{Matching DEM/Satellite Image}

In all test cases, the shaded model of the respective DEM was used as reference image and the IKONOS image was matched to it.
The used intensity based matching algorithm is described in [1] and [5] and was originally developed for MEOSS and MOMS-2P 3-line alongtrack stereo scanner imagery. Promising matching points are selected in the reference image using the Förstner operator and the second image is searched for the corresponding points using cross correlation. The resulting matches are then refined to subpixel accuracy by applying a local least squares matching. The algorithm returns quality parameters for each matching point.

In a second matching, also the Camri matching was used. Therefore, a regular point grid is created in the reference image and the points are matched to the second image based on entropy parameters. The Camri matching is described in [6]. As this method is based on mutual information (MI), a larger image area, here $500 \times 500$ pixel surrounding one point is accounted for. This makes it a more promising method than the intensity based matching.

\section{RESULTS}

\subsection{DEM created from IKONOS stereo pair}

As this DEM is the one generated from the data with the highest resolution, it is also the most detailed one. Therefore it is expected to deliver the best local (detailed) results.

Using the intensity based matching, 20 conjugate points were found with a correlation coefficient higher than 0.9 . Optical inspection of the matched points in both images reveals reasonable matches. Figure 2 shows one of the matching points in both images.

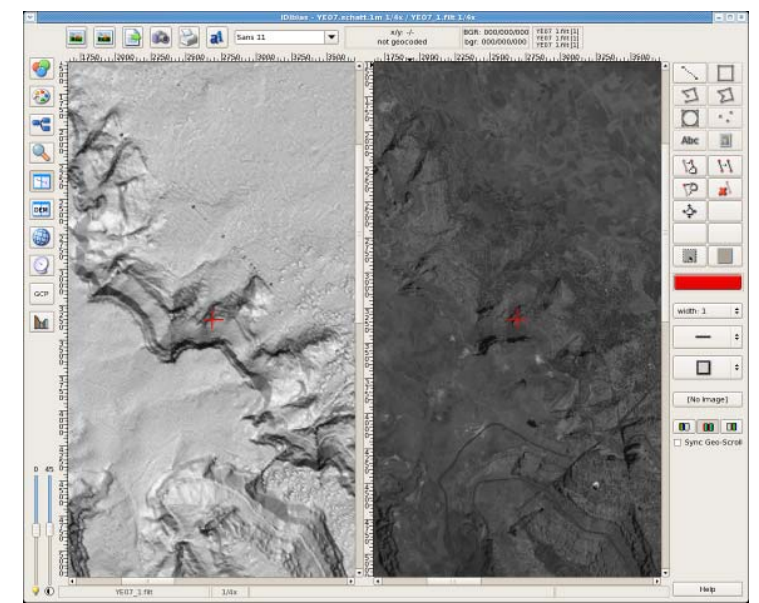

Figure 2: Conjugate point in shaded model of IKONOS DEM on the left and IKONOS image on the right.

Thus the intensity based matching returns a sufficient number of GCPs to correct the RPCs of the image and achieve a better coregistration of 
image and DEM. With the Camri matching, of 110 input points 60 were found in the IKONOS image. Mismatches could not be identified with visual inspection.

\subsection{DEM created from ALOS/PRISM triplet}

The DEM generated from the ALOS/PRISM data is less detailed than the one generated from the IKONOS stereo pair. Not only the resolution of the imagery is lower, also the radiometric characteristics of PRISM images are worse than that of the IKONOS scenes. Therefore, in the point clouds there are more and bigger gaps so that it is partly necessary to interpolate over large areas. Accounting for these constraints, the results of these tests are expected to be worse than the ones of the tests using the IKONOS-DEM.

Using the intensity based matching, only 5 conjugate points were found with a correlation coefficient higher than 0.9. When lowering this threshold to $0.8,32$ points were found. Optical inspection shows that about one third of the points are mismatches, however most of the matched points look reasonable, e.g. as shown in figure 3.

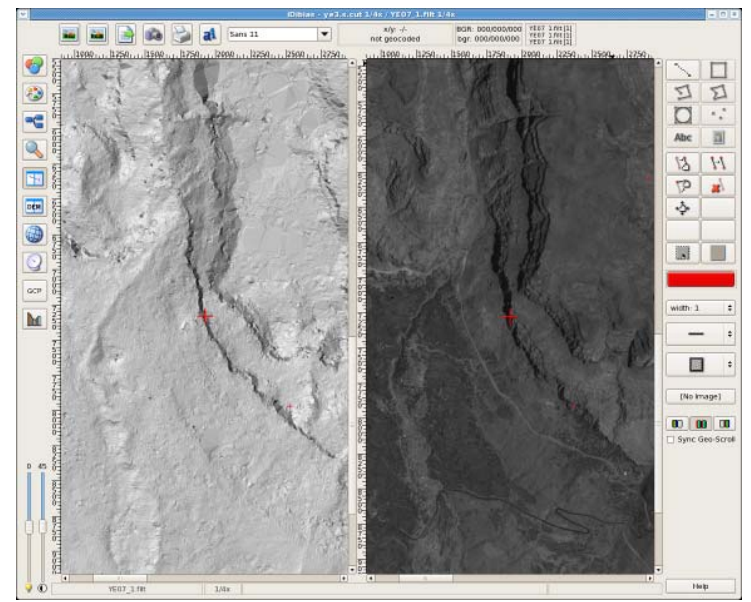

Figure 3: Conjugate point in shaded model of ALOS/PRISM DEM on the left and IKONOS image on the right.

The matching results could be improved by improving the ALSO/PRISM DEM generation. This is currently investigated [7]. With the Camri matching, of 110 input points 56 were found in the IKONOS image and the results look very reasonable.

\subsection{SRTM-DEM}

Since the resolution of the SRTM-DEM is much lower than the resolution of the IKONOS image, and thus the level of detail in the SRTM DEM is much lower than in the IKONOS image, here it is doubtful to find any matching points.
In the test using the intensity based matching, no conjugate points were found. With the Camri matching, of 110 input points 33 were found in the IKONOS image. The results look reasonable; however, there are also some doubtful matches. These matches are mainly in flat area, where the entropy is very low which corresponds to agricultural area in the IKONOS image with also low entropy. Nevertheless, there are still a sufficient number of conjugate points left to correct the RPCs and thus improve the coregistration of the IKONOS image to the SRTM DEM.

\section{APPLICATION EXAMPLE}

For a change detection investigation, it was necessary to coregister two IKONOS orthoimages. Therefore, a matching was performed with the orthoimages and at the conjugate points, the displacements were calculated. However, since no GCPs were available, most displacements were due to the bad coregistration. Also after a matching of both raw IKONOS images and a relative RPC correction, there were still terrain induced displacements in the orthoimages. This was due to the fact that the images were coregistered to each other, but not to the DEM, created from an IKONOS stereo pair, that was used to create the orthoimages. Figure 4 shows a plot of the displacements in one section of the images.

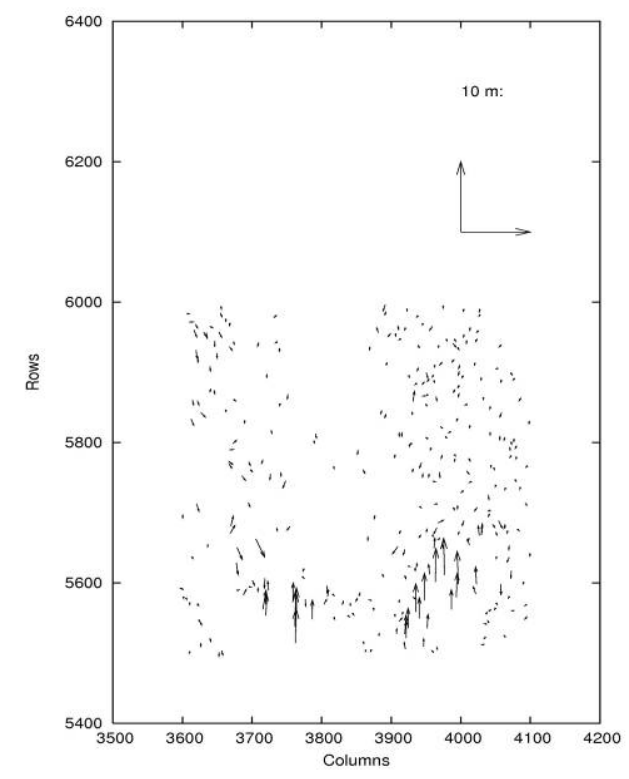

Figure 4: Displacements calculated from a matching of two IKONOS images of the test area in Yemen. The bigger displacements in the lower part are clearly induced by the bad coregistration of the images to the DEM.

To improve this coregistration, the intensity based matching method described above was used 
to find some GCPs in the DEM. The RPCs were corrected using the obtained 20 GCPs and again the orthoimages were generated. After another matching, the results were much better. Figure 5 shows that both the number and the size of the displacements decreased strongly. Similar results are obtained when using the Camri matching method.

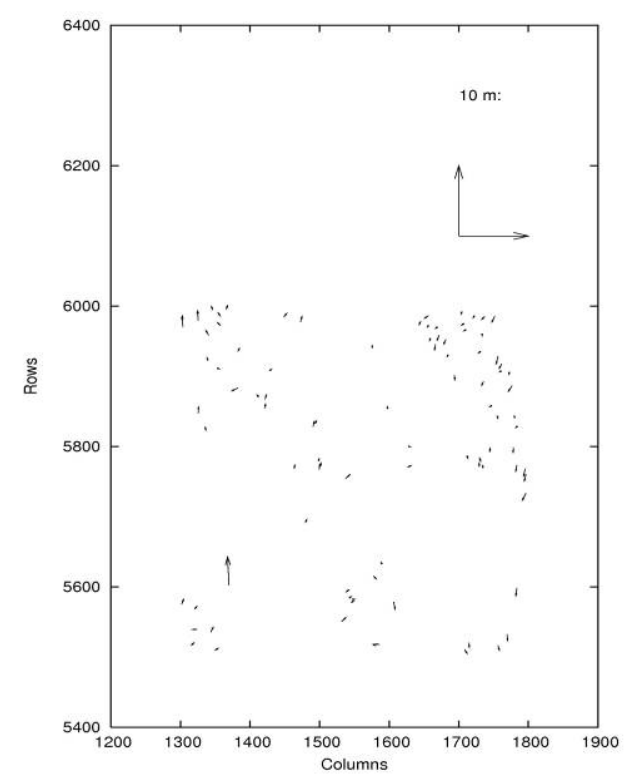

Figure 5: Displacements calculated from a matching of two IKONOS images of the test area in Yemen after correcting the RPCs with GCPs derived from DEM/image matching as described above.

\section{CONCLUSION}

It has been shown that shaded models of DEMs can serve as a source for GCPs if no other ground truth is available. Two different matching methods have been evaluated on three DEMs with different resolution and therefore different levels of detail. The hierarchical intensity based matching returns good results if DEM and satellite image have a similar resolution. However, it failed for the test case using SRTM DEM as reference image and an IKONOS scene as second image. The mutual information based Camri matching on the other hand returns a sufficient number of good matching points to allow for a RPC correction in all three test cases.

Unfortunately, for the test area no independent GCPs were available for a quantitative evaluation. This has to be done in the future in other test areas. However, the application example in chapter 5 shows the potential of this method. Since the absolute accuracy of the GCPs depends on the accuracy of the used DEM, absolute pixel or even subpixel accuracy may not be achieved in case of very high resolution images. However, a sufficient accurate coregistration of different images to a DEM can be realized which is necessary e.g. for change detection purposes.

Accounting for the nearly global availability of the SRTM DEM, the increasing availability of stereo satellite imagery like ALOS/PRISM or Cartosat and planned mission like Tandem-X, a source for GCPs could be available for almost every place on the earth. However, the test area used in this paper is a rural arid area, which is well suited for automatic image matching. In further investigations, the method has to be evaluated in less ideal areas, i.e. urban or forested areas. Nevertheless, the results presented in this paper are very promising.

\section{REFERENCES}

[1] M. Lehner, R. S. Gill (1992): "Semi-automatic derivation of digital elevation models from stereoscopic 3-line scanner data“, IAPRS Vol. 29 part B4, Washington, USA, pp 68-75, 1992

[2] S. Leprince, S. Barbot, F. Ayoub, J. P. Avouac (2007): "Automatic and Precise Orthorectification, Coregistration and Subpixel Correlation of Satellite Images, Application to Ground Deformation Measurements”, IEEE Transactions on Geoscience and Remote Sensing, Vol. 45, No. 6; 2007

[3] Schneider, Mathias und Lehner, Manfred und Müller, Rupert und Reinartz, Peter (2009): Stereo Evaluation of ALOS/PRISM Data on ESA-AO Test Sites - First DLR Results. In: ALOS PI Symposium 2008, 2008-11-03 - 2008-11-07, Rhodes (Greece).

[4] M. Lehner, R. Müller, P. Reinartz (2005): “DSM and Orthoimages from QuickBird and Ikonos Data using Rational Polynomial Functions”; Heipke, C.; Jacobsen, K.; Gerke, M. [Eds.]: High Resolution Earth Imaging for Geospatial Information, IAPRS, Vol. XXXVI, S. CDROM -, High Resolution Earth Imaging for Geospatial Information, Hannover, 17.-20. Mai 2005, ISBN ISSN No. 1682-1777; 2005

[5] W. Kornus, M. Lehner, M. Schroeder (2000): "Geometric inflight calibration by block adjustment using MOMS-2P 3-line-imagery of three intersecting stereostrips”; SFPT (Société Francaise de Photogrammétrie et Télédétection), Bulletin Nr. 159, pp. 42-54; 2000

[6] H. Chen, P.K. Varshney, and M.K. Arora (2003): "Mutual information based image registration for remote sensing data", International Journal of Remote Sensing, vol. 24, no 18, pp. 3701-3706, 2003

[7] R. Müller, M. Schneider, P. Reinartz, F. Schwonke (2009): „Stereo evaluation of ALOS PRISM and IKONOS data in Yemen"; to be published in IGARSS09 conference proceedings; 2009

[8] R. Müller, T. Krauß, M. Lehner, P. Reinartz, (2007): Automatic Production Of A European Orthoimage Coverage Within The GMES Land Fast Track Service Using Spot 4/5 And Irs-P6 Liss III Data, ISPRS Conference Proceedings, Volume XXXXVI, ISPRS Workshop Hannover, Germany, May 2007, 6 p. 